\title{
Electron Density and Dielectric Properties of Highly Porous MOFs: Binding and Mobility of Guest Molecules in $\mathrm{Cu}_{3}(\mathrm{BTC})_{2}$ and $\mathrm{Zn}_{3}(\mathrm{BTC})_{2}$
}

\author{
Rebecca Scatena, ${ }^{*}{ }^{\dagger}$ Yannick T. Guntern, ${ }^{\dagger, \S}$ and Piero Macchi ${ }^{*}, \dagger, \ddagger$ \\ ${ }^{\dagger}$ Department for Chemistry and Biochemistry, University of Bern, Freiestrasse 3, Bern 3012, Switzerland \\ ${ }^{\ddagger}$ Department of Chemistry, Materials and Chemical Engineering, Polytechnic of Milan, via Mancinelli 7, Milano 20131, Italy
}

\section{Supporting Information}

ABSTRACT: Two isostructural highly porous metal-organic frameworks, the wellknown $\left\{\mathrm{Cu}_{3}(\mathrm{BTC})_{2}\right\}_{n}(\mathrm{BTC}=1,3,5$-benzenetricarboxylate), often appointed with the name HKUST-1, and $\left\{\mathrm{Zn}_{3}(\mathrm{BTC})_{2}\right\}_{n}$, have been investigated as models for the buildup of dielectric properties, differentiating the role of chemi- and physisorbed guest molecules and that of specific intraframework and framework-guest linkages. For this purpose, electron charge density analysis, impedance spectroscopy, density functional theory simulations, and atomic partitioning of the polarizabilities have been exploited. These analyses at different degrees of pores filling enabled one to observe structural and electronic changes induced by guest molecules, especially when chemisorbed. The electrostatic potential inside the pores allows one to describe the absorption mechanism and to estimate the polarization of guests induced by the framework. The dielectric constant shows very diverse frequency dependence and magnitude of real and imaginary components as a consequence of (I) capture of guest molecules in

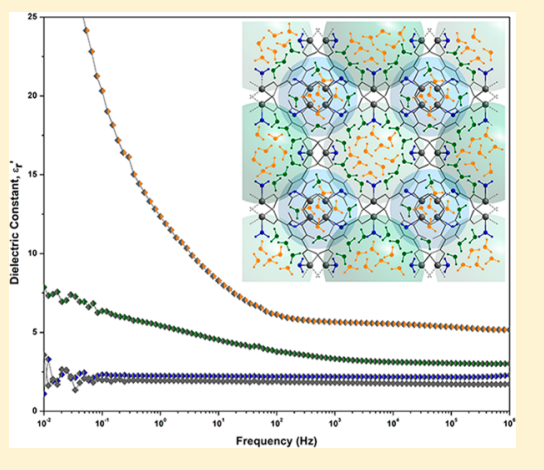
the pores during synthesis, (II) MOF activation, and (III) water absorption from the atmosphere after activation. Comparison with calculated static-dielectric constant and atomic polarizabilities of the material has allowed for evaluating building blocks' contribution to the overall property, paving the way for reverse crystal engineering of these species.

\section{INTRODUCTION}

According to Bersuker's definition, ${ }^{1}$ material science is "the translation from microscopic foundations of matter to macroscopic performance". The central paradigm of material science is the structure-property relationship, which implies that a specific functionality is activated (or quenched) depending on both nature and arrangement of atoms, molecules, and the interactions among them. At the nanoscale level, these interactions are not governed by classical physics, but by quantum mechanics. In fact, they depend on the distribution and flow of electrons in the field generated by their mutual interplay and the interplay with the nuclei. The field may also be modified externally, for example, in capacitors.

The modern microelectronic industry is seeking the downscaling of integrated circuits to enhance the performances of devices. In this context, there is a huge need for highly efficient electric insulators. This implies moving from traditional silica-based materials to air gap ones. ${ }^{2}$ The fundamental idea is to build a structure around the lowest possible dielectric constant $\left(\varepsilon_{\mathrm{r}}\right)$ medium, which is vacuum (or air). In this respect, materials featuring large pores and poorly correlated scaffolding may serve this purpose. This description seamlessly fits with that of metal-organic frameworks (MOFs), ${ }^{3}$ materials known for more than a couple of decades and nowadays fabricated with simple and low-cost processes. ${ }^{4,5}$ Further attention on this class of materials is driven by the countless available types of network topologies, ${ }^{6}$ which enable the finetuning of many properties. ${ }^{7}$ Their porous structures and the fact that light atoms $(\mathrm{C}, \mathrm{H}, \mathrm{N}$, and $\mathrm{O})$ mainly constitute them infer very low mass (and electron) densities that typically result in low electric susceptibility as well. In fact, according to rough estimations of static- $\varepsilon_{\mathrm{r}}$, MOFs are very promising next generation interlayer materials. $^{8}$

Despite this enormous potential, only some studies have been reported ${ }^{9-19}$ so far on experimental measurements of dielectric constant of MOFs. The observations concern materials featuring different topologies and compositions. Moreover, they refer to different frequency ranges and conditions such as temperature and atmosphere. The same holds true for first-principles simulations, ${ }^{20-22}$ which may give more insight in the effective buildup of a property. This lack of systematic data does not allow much rationalization and therefore theorization of an optimal structure-property correlation.

Having this in mind, we have started a project with the aim of investigating how atomic and electronic structures of MOFs may affect the dielectric properties featured by these materials. For this investigation, we selected two isostructural MOFs, $\left\{\mathrm{Cu}_{3}(\mathrm{BTC})_{2}\right\}_{n} \quad(\mathbf{1} \text {, also known as HKUST-1 })^{23}$ and $\left\{\mathrm{Zn}_{3}(\mathrm{BTC})_{2}\right\}_{n}(2)$, where BTC $=1,3,5$-benzenetricarboxylate. They offer a variety of variables that may be changed to monitor their effect on the dielectric behavior. In addition, we were motivated by the low refractive index reported for a

Received: April 4, 2019

Published: May 25, 2019 
surface-anchored thin film of $\mathbf{1}$ in the visible light. ${ }^{24}$ We are interested to analyze bulk properties over a wider range of frequencies, in particular lower frequencies for potential electronics applications, and to assess the key factors that may tune or trigger them. Our strategy consists of investigating the sequence from atomic structure to dielectric function through the electron density distribution and vice versa. The parameters to be varied are, for example: (a) geometries of the building blocks; (b) connectivity at metal centers (with possible ancillary ligands that may deactivate the framework); (c) different kinds of sorption (chemi- or physi-sorption) on the internal surfaces and in the channels of the MOFs; and (d) stereoelectronic differences between an open-shell and a closed-shell metal ion.

The contribution of the building blocks to the overall macroscopic properties is disentangled by characterizing the bonding properties and estimating the electrostatic forces. To obtain this information, quantum crystallographic studies are necessary. These include the determination the material polarizability from impedance spectroscopy, which then needs to be coupled with first principle simulations to partition the electron density and dielectric polarizability functions in terms of the constituting building blocks.

The experimental determination of accurate electron density distribution in highly porous MOFs has been hampered by the poor quality of the crystals that do not guarantee the sufficient X-ray diffraction necessary for the goal. As a matter of fact, only charge density studies in nonporous metal organic materials are known. ${ }^{25-28}$ For this reason, in this study, we coupled a careful X-ray structural analysis as a function of pores filling with density functional theory (DFT) simulation of the electron charge density distribution.

\section{EXPERIMENTAL SECTION}

Synthesis and Crystal Growth. All chemicals were purchased commercially and were used as received without further purification. The syntheses were performed in $50 \mathrm{~mL}$ volume Teflon-lined stainless-steel autoclave reactors. Octahedral single crystals (exposing the 111 face) and microcrystalline powder of $\left[\mathrm{Cu}_{3}(\mathrm{BTC})_{2}\right]_{n}(\mathbf{1})$ were prepared according to the $\mathrm{Wu}$ et al. ${ }^{29}$ strategy. Single crystals with a size up to $300 \mu \mathrm{m}$ were grown by increasing the cooling time up to 12 h. Cubic single crystals (exposing the 100 face) of $\left[\mathrm{Zn}_{3}(\mathrm{BTC})_{2}\right]_{n}(2)$ were obtained by using the synthesis reported by Feldblyum et al.; ${ }^{30}$ the size of single crystals was increased up to $250 \mu \mathrm{m}$ by using 3 times the reagent's concentration. The single crystals were washed by decanting three times with $60{ }^{\circ} \mathrm{C}$ DMF and stored in $\mathrm{Cl}_{3} \mathrm{CH}$.

MOF Activation. Microcrystalline pellets of 1 were activated by heating to $200{ }^{\circ} \mathrm{C}$ for $20 \mathrm{~h}$ at $10^{-3} \mathrm{mbar}$. As was reported previously by Schlichte et al., ${ }^{31}$ this treatment retrieves a complete removal of guest molecules. Thermal gravimetric analysis (TGA) agrees with this scenario; see the Supporting Information. Activated pellets for impedance spectroscopy were loaded into the sample holder by working in a glovebox with $\mathrm{N}_{2}$ atmosphere and ca. 5 ppm of $\mathrm{H}_{2} \mathrm{O}$. More gentle activation treatments were tested on single crystals to retain high single-crystal quality: (a) Heat. A crystal of $\mathbf{1}$ was heated to $373 \mathrm{~K}$ under the $\mathrm{N}_{2}$ flow of the cryostat for $3 \mathrm{~h}$. The activated crystal was in situ cooled to $110 \mathrm{~K}$, and an X-ray data set was collected. (b) Vacuum and heat. Crystals of $\mathbf{1}$ were heated in a Shlenk flask to $373 \mathrm{~K}$ upon vacuum $\left(10^{-2} \mathrm{mbar}\right)$ for $1.5 \mathrm{~h}$; the temperature was further increased to $393 \mathrm{~K}$ for $1 \mathrm{~h}$ and then cooled naturally to room temperature. To prevent rehydration, the crystals were covered with Paratone $\mathrm{N}$ oil before exposing them to air. (c) Crystals of 1 were heated in a Büchi oven to $373 \mathrm{~K}$ upon vacuum $\left(10^{-2} \mathrm{mbar}\right)$. The temperature was increased in ca. $6 \mathrm{~h}$ to $473 \mathrm{~K}$ and then kept at $473 \mathrm{~K}$ for $12 \mathrm{~h}$. Last, the crystals were cooled naturally to room temperature in a glovebox with $\mathrm{N}_{2}$ atmosphere and covered with Paratone $\mathrm{N}$ oil.
Dielectric Properties. The measurements were carried out with a Solartron impedance/Gain-Phase analyzer ModulabXM equipped with XM MFRA $1 \mathrm{MHz}$ and XM MAT $1 \mathrm{MHz}$ control modules. A 12962 A sample holder was used with dried powder pellet samples with a diameter of $25 \mathrm{~mm}$ prepared by applying a pressure of $0.2 \mathrm{GPa}$. The sample holder consists of a two brass parallel electrodes capacitor with a $20 \mathrm{~mm}$ diameter active area. It is provided with a guard ring, which, together with a sample diameter bigger than the active electrode surface, reduces the fringing effect of stray fields at the edge of tested materials. The sample holder was set in a glovebox with $\mathrm{N}_{2}$ atmosphere to ensure dry measurement conditions. The measurement parameters were controlled with the ModulabXM software. The measurement setup consists of a fixed mode generator voltage level of $0 \mathrm{~V}$ with amplitude of $100 \mathrm{mV}$ and a frequency sweep from $10 \mathrm{mHz}$ to $1 \mathrm{MHz}$. The electronic field is applied between the two electrodes, across a measured thickness of the sample, at room temperature. Real $\left(\varepsilon_{\mathrm{r}}^{\prime}\right)$ and imaginary $\left(\varepsilon_{\mathrm{r}}^{\prime \prime}\right)$ components of $\varepsilon_{\mathrm{r}}$ were obtained from frequency-dependent impedance measurements.

X-ray Data Collections and Structural Refinement. Crystals were glued on a glass fiber, and the temperature was set by using an Oxford Cryostream flow cryostat. Extensive high-resolution data collections $\left(d_{\min }=0.50 \AA\right)$ were performed at $110.0(2) \mathrm{K}$ for compound 1 subjected to different activation treatments. All measurements were made on an Oxford Diffraction SuperNova area-detector diffractometer using mirror optics, monochromated, and Al filtered ${ }^{32}$ microsource Mo $K_{\alpha}$ radiation $(\lambda=0.71073 \AA)$. Data reductions were performed using CrysAlisPro software. ${ }^{33}$ Intensities were analytically corrected for Lorentz and polarization effects, and for absorption with the Gaussian method based on the crystal shape. The conventional structural refinement was carried out with SHELXL. ${ }^{34}$ Non-hydrogen atoms were refined with anisotropic displacement parameters. The hydrogen atom of the BTC aromatic ring was placed in calculated positions with isotropic displacement parameters set to $1.2 \times U_{\text {eq }}$. The residual solvent molecules are highly disordered, and attempts to locate and refine the solvent peaks were unsuccessful. Single crystal subjected to different activation strategies features residuals at apical positions of the paddlewheel as well as in the center of the pores. The latter being high symmetry sites are "attractors" of residual density. ${ }^{35-37}$ These residuals do not affect the description of the electronic state in the framework. On the other hand, the residuals close to the paddlewheels are more effectively a sign of a fraction of guest molecules still coordinated to the framework and by refining the site occupancy of the oxygen atom of the coordinated apical molecule a chemisorption degree (CS) was estimated. The atomic displacement parameter (ADP) was constrained to the result refined from data for a crystal stored in $\mathrm{MeOH} /$ $\mathrm{H}_{2} \mathrm{O}$ after synthesis, assuming in that case a $100 \%$ occupancy. Selected crystallographic data and refinement details are summarized in the Table SI 1 .

DFT Simulations. Periodic Density Functional Theory ( $\mathrm{p}-\mathrm{DFT})$ calculations of the electron density distribution, geometry optimizations, and static dielectric constant were carried out by using CRYSTAL $14^{38}$ software. The functional B3LYP was used with basis pob-TZVP. ${ }^{39}$ The static dielectric tensor was computed with the coupled Perturbed Kohn-Sham method as implemented in CRYSTAL14. ${ }^{38}$ Gas-phase DFT calculations of the electron density distribution, geometry optimization, and molecular polarizability upon external electric field on $\left[\mathrm{M}_{2}\left(\mathrm{H}_{2} \mathrm{BTC}\right)_{4}\left(\mathrm{H}_{2} \mathrm{O}\right)_{2}\right]$, $\left[\mathrm{M}_{2}\left(\mathrm{H}_{2} \mathrm{BTC}\right)_{4}\left(\mathrm{H}_{2} \mathrm{O}\right)\right]$, or $\left[\mathrm{M}_{2}\left(\mathrm{H}_{2} \mathrm{BTC}\right)_{4}\right]$ fragments were carried out with Gaussian 09 code. ${ }^{40}$ The cluster $\left[\mathrm{M}_{2}\left(\mathrm{H}_{2} \mathrm{BTC}\right)_{4}\left(\mathrm{H}_{2} \mathrm{O}\right)_{2}\right]$ with dipole moments of value one or one-half $\mathrm{H}_{2} \mathrm{O}$ molecule in place of the coordinated $\mathrm{H}_{2} \mathrm{O}$ molecules was used to optimize the paddlewheel geometry upon the only electrostatic effect of apical coordination at $50 \%$ or $100 \%$ of chemisorption degree, respectively. The B3LYP functional was employed with the basis sets pob-TZVP, ${ }^{39}$ 6$31 \lg (2 \mathrm{~d}, 2 \mathrm{p})$, and $6-311++\mathrm{g}(2 \mathrm{~d}, 2 \mathrm{p})$. The topological analysis of the electron density was performed with AIMAll software. ${ }^{41}$ PolaBer software $^{42}$ was employed to calculate and project atomic polarizabilities. The strength of the coordinative interactions between the metal paddlewheel and the ligands was estimated from the difference 
between the energy of optimized fragments and their assembly. The energy for the apical interaction between the paddlewheel and the apical $\mathrm{H}_{2} \mathrm{O}$ molecule was estimated by in vacuo or periodic DFT calculations at the levels of theory described before, whereas only in vacuo DFT calculations were employed to estimate binding energy between a carboxylate group and paddlewheel. The basis set superposition error was estimated by counterpoise correction as implemented in Gaussian 09.

\section{RESULTS AND DISCUSSION}

Compounds $\mathbf{1}$ and $\mathbf{2}$ are isostructural and crystallize in the cubic space group $F m \overline{3} m$. The three-dimensional periodic structure is generated by binuclear $\mathrm{M}$ (II) paddlewheel secondary building units (SBUs) connected via the tritopic 1,3,5-benzenetricarboxylate organic linker. The framework features two kinds of intersecting pores with diameters of about 10 and $15 \AA$ (cyan and green spheres, respectively; see Figure 1). The theoretical porosity amounts to $69 \%$ of the

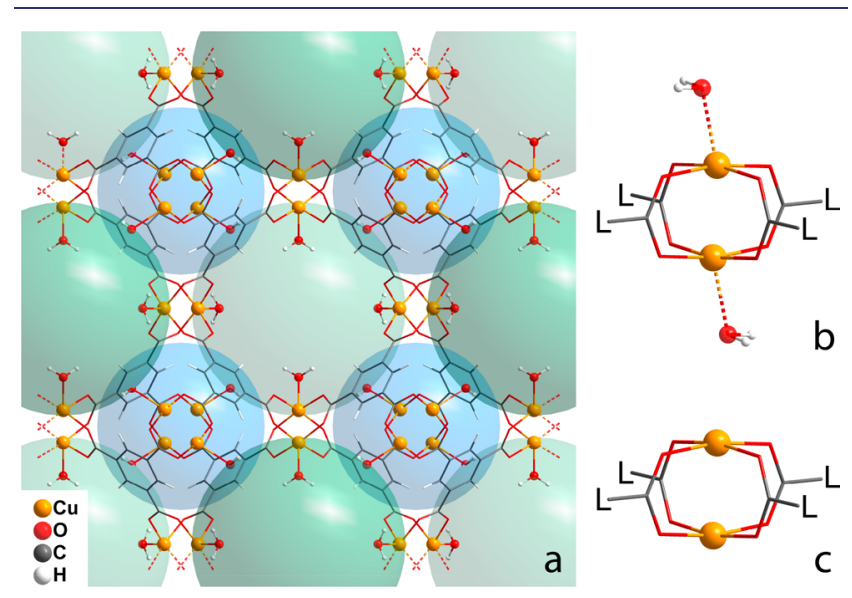

Figure 1. Optimized crystal structure of $\left\{\mathrm{Cu}_{3}(\mathrm{BTC})_{2}\left(\mathrm{H}_{2} \mathrm{O}\right)_{3}\right\}_{n}$ : (a) unit cell content, intersecting pores are depicted as cyan and green spheres, (b) paddlewheel with apical ligands, and (c) paddlewheel without apical ligands.

volume as calculated with a probe radius of $1.2 \AA .^{43}$ However, as-synthesized, the samples contain a large amount of solvent molecules, hosted in the pores. Moreover, the M(II) ions expand their coordination attaching an additional ligand, opposed to the $\mathrm{M}-\mathrm{M}$ bond; see Figure $1 \mathrm{~b}$. The nature of the linkage between guest molecules and host framework as well as the role of guest molecules in modifying materials properties have not been deeply investigated despite the paramount importance and notoriety of this class of MOFs. An atomresolved microscope able to protrude inside the pores is not available, and therefore those main questions must be answered indirectly by the analysis of bulk electronic properties, average features of the atomic and electronic structures, as well as through simulations.

Finely milled powders of 1 lose completely guest molecules at $200{ }^{\circ} \mathrm{C}^{31}$ If the sample is heated in a vacuum of $10^{-3} \mathrm{mbar}$, powder X-ray diffraction shows that the characteristic peaks of the hydrated samples disappear only at $200{ }^{\circ} \mathrm{C}$, in agreement with thermogravimetric analysis (TGA), which presents two sharp steps connected to solvent loss and decomposition of the MOF, respectively (see the Supporting Information). On the other hand, isothermal-TGA at $100{ }^{\circ} \mathrm{C}$ indicates that the activation is only partial at this temperature. In fact, in this case, an additional step also due to solvent loss appears when the temperature is further increased. The hygroscopic behavior of the compound is evident because a sample exposed to air after activation undergoes new losses of guest molecules in the TGA before reaching again the activated condition. The guest molecules trapped in $\mathbf{1}$ during or after the synthesis, $\mathrm{H}_{2} \mathrm{O}$ and $\mathrm{MeOH}$, are volatile, whereas $\mathbf{2}$ is synthesized in DMF, which is a high-boiling solvent. Consequently, decomposition of the framework 2 occurs before reaching complete activation. In addition, as it has been reported, ${ }^{30} 2$ suffers from surface instability, which prevents high degrees of activations because of pores closure at the surface. Transmetalation, that is, the exchange of metal ions in the framework when $\mathbf{2}$ is immersed in a solution of $\mathrm{Cu}(\mathrm{II})$, is also compatible with a lower stability of the zinc based SBU. ${ }^{44}$ The instability and the activation limits of 2 prevent its experimental investigation and potential application. However, the structural analogy between 1 and 2 allows, through ab initio simulations, one to link stereoelectronic configurations with dielectric properties and obtain useful comparative information.

Given the structure of the MOFs, guest molecules can easily enter both kinds of pores and find three possible accommodations: (a) inside the pores without any linkage to the MOF and featuring a free movement; (b) adsorption on the internal surfaces; and (c) weak coordination to the metal centers. The different kinds of sorption must be recognizable in the dielectric constant of the solid, especially when molecules are polar and polarizable, dealing with different mobility of the guest dipoles. The presence of guest molecules may also affect the framework component of the dielectric constant. Therefore, impedance spectroscopy over a broad frequency range was used to measure the complex dielectric constant (real $\varepsilon_{\mathrm{r}}{ }^{\prime}$ and imaginary $\varepsilon_{\mathrm{r}}{ }^{\prime \prime}$ ) on pellets of 1 , subjected to different treatments and in different atmospheres (Figure 2).

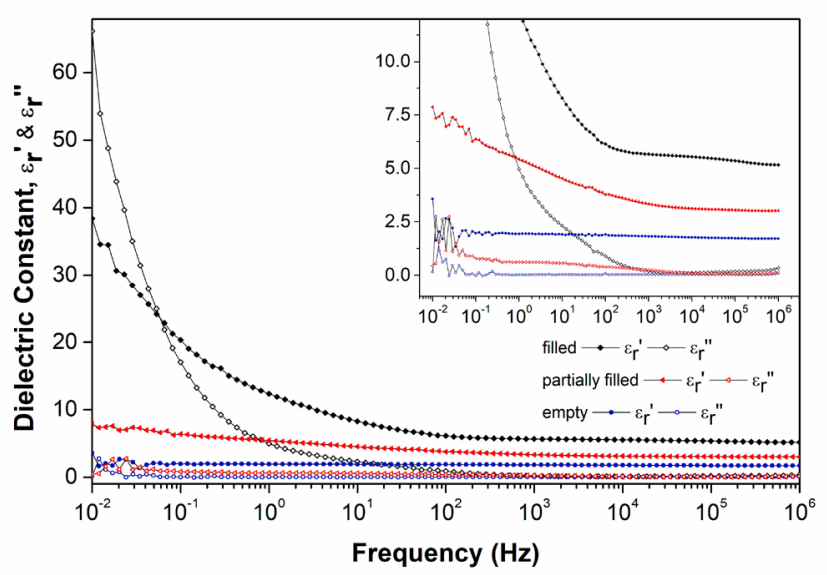

Figure 2. Real $\left(\varepsilon_{\mathrm{r}}{ }^{\prime}\right)$ and imaginary $\left(\varepsilon_{\mathrm{r}}{ }^{\prime \prime}\right)$ dielectric constant for $\mathbf{1}$ assynthesized (filled with $\mathrm{H}_{2} \mathrm{O}$ and $\mathrm{MeOH}$ ), measured in air after activation (partially filled with $\mathrm{H}_{2} \mathrm{O}$ ), and measured in $\mathrm{N}_{2}$ after activation (empty). The inset highlights the differences between activated samples measured in different atmospheres.

In the range of frequencies investigated $\left(10^{-2}-10^{6} \mathrm{~Hz}\right)$, several mechanisms may contribute to the dielectric constant, such as electric polarization, as well as nuclear and ionic displacements (implying reorientation of permanent dipoles or ion migration). Instead, electronic absorption occurs at higher frequencies, and it is of course not relevant for our investigation. 
When $\mathbf{1}$ is activated and measured in $\mathrm{N}_{2}$ atmosphere, $\varepsilon_{\mathrm{r}}{ }^{\prime}$ is low and remarkably stable against frequency. Under these conditions, the MOF appears to be an empty object. Noteworthy, the imaginary component $\varepsilon_{\mathrm{r}}{ }^{\prime \prime}$ is close to zero, indicating no mobility of ions or dipoles in the entire structure. On the contrary, when the sample is filled (like assynthesized), the guest molecules induce a significant dependence of $\varepsilon_{\mathrm{r}}$ from the frequency; moreover, $\varepsilon_{\mathrm{r}}{ }^{\prime \prime}$ is very large and it even exceeds $\varepsilon_{\mathrm{r}}{ }^{\prime}$ below $0.1 \mathrm{~Hz}$. This trend is symptomatic of a relevant energy loss due to friction among mobile molecules. Such a contribution arises from polar guest molecules $\left(\mathrm{H}_{2} \mathrm{O}\right.$ or $\mathrm{MeOH})$ that are not directly attached to the framework surface and easily move inside the pores. Differently, the $\varepsilon_{\mathrm{r}}{ }^{\prime \prime}$ of an activated sample measured in air does not overwhelm $\varepsilon_{\mathrm{r}}{ }^{\prime}$ at low frequencies, addressing guest molecules that are less free to reorient their dipoles upon application of the oscillating field. This scenario is produced by the hygroscopic character of $\mathbf{1}$, which causes a partial adsorption of $\mathrm{H}_{2} \mathrm{O}$ molecules. The adsorption first concerns molecules, which are more strongly interacting with the framework and therefore less movable. The different amount of guest molecules in these two cases (nonactivated sample or activated and then partially rehydrated) emerges also from the high frequency limit of $\varepsilon_{\mathrm{r}}{ }^{\prime}$, reflecting the electronic polarization only. In fact, at $1 \mathrm{MHz}$ $\varepsilon_{\mathrm{r}}^{\prime}$ is $5.163(8)$ and $3.017(4)$, for filled and partially filled samples, respectively, speaking for higher and lower density of the bulk (hence different content of guest molecules). For comparison, the fully activated material has a much lower dielectric constant at high frequency $(1.717(3))$, which does not change with frequency, but for the small contribution of the nuclear polarization of atoms belonging to the framework, that adds up slowly at frequencies below $0.1 \mathrm{MHz}$. In keeping with this interpretation, the DFT calculation of the static dielectric tensor (i.e., neglecting nuclear displacements) for the empty framework seamlessly matches the experimental value $\left(\varepsilon_{\mathrm{r}, \text { calc }}=1.74\right.$ vs $\left.\varepsilon_{\mathrm{r} \text {,expt }}=1.717(3)\right)$. As discussed above, compound $\mathbf{2}$ is not measurable in the activated state; therefore, no comparison is possible, unless using the theoretical calculations that predict an even lower static- $\varepsilon_{\mathrm{r}}{ }^{\prime}$ (1.49); see Table 1. This is expected due to the inherently lower polarizability of a closed-shell cation like $\mathrm{Zn}^{2+}$, as compared to $\mathrm{Cu}^{2+}$.

Table 1. Calculated Static Dielectric Constants for 1 and 2, and Atomic Polarizabilities of the Metal Ions

\begin{tabular}{lcc}
\multicolumn{1}{c}{ MOF } & static- $\varepsilon_{\mathrm{r}}$ & $\alpha_{\mathrm{M}_{\text {IISO }}}\left(\right.$ bohr $\left.^{3}\right)$ \\
$\left\{\mathrm{Cu}_{3}(\mathrm{BTC})_{2}\right\}_{n}$ & 1.74 & 14.592 \\
$\left\{\left[\mathrm{Cu}_{3}(\mathrm{BTC})_{2}\right]\left(\mathrm{H}_{2} \mathrm{O}\right)_{3}\right\}_{n}$ & 1.80 & 14.386 \\
$\left\{\mathrm{Zn}_{3}(\mathrm{BTC})_{2}\right\}_{n}$ & 1.49 & 9.162 \\
$\left\{\left[\mathrm{Zn}_{3}(\mathrm{BTC})_{2}\right]\left(\mathrm{H}_{2} \mathrm{O}\right)_{3}\right\}_{n}$ & 1.52 & 9.643 \\
\hline
\end{tabular}

Three different regimes in the pore-filling process were identified from the dielectric constant measurements: (a) empty MOF (or activated), showing no dipole-mobility and therefore no sign of mobile guest molecules; (b) partially filled due to partial rehydration after the activation, showing some dipole-reorientation and higher density; and (c) filled, when no-activation of the sites has been attempted, showing larger dielectric constant and dipole-reorientation (Figure 3). This analysis allows also one to appreciate that different fillings differ not only in the amount of guest molecules trapped, but also in the nature of their binding to the framework.

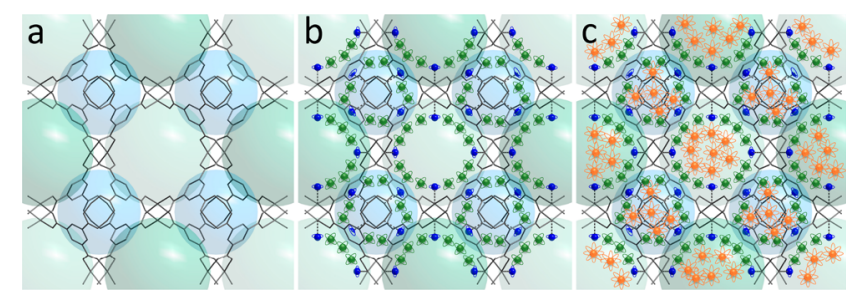

Figure 3. Schematic representation of the porous MOF with different degrees of filling: (a) empty, (b) partially filled, and (c) filled. The vibrational and rotational degrees of freedom increase going in the series: blue, green, and orange.

Impedance spectroscopy, however, does not establish whether the dielectric contributions of guest molecules and MOF are simply additive, or if they correlate. High-resolution structural measurements on $\mathbf{1}$ at different degrees of guest sorption and theoretical electron charge density modeling may give more insight into intra- and intermolecular interactions. This approach requires high quality and large size of the crystal samples.

Various activation procedures were tested on single crystals to remove the guest molecules. Harsh conditions (very high temperature and vacuum) induce a too rapid activation and lead to severe damage of the samples, which break into polycrystalline material. It was possible to retain rather high crystallinity upon activation by using slow temperature gradients and lower vacuum. These treatments partially remove the molecules coordinated to the paddlewheel node and do not allow selective discrimination among different guests, the coordinated (chemisorbed), the physisorbed, and the movable (filling) molecules. While 1 showed a remarkable single-crystal stability with high-resolution $(d=0.5 \AA)$ X-ray scattering, the isostructural 2 based on the $\mathrm{Zn}$ (II) paddlewheel node turned polycrystalline under any activation condition, even if mild. Noteworthy, $\mathbf{1}$ is the only example reported in the Cambridge Structural Database ${ }^{45}$ (CSD) in which the copper paddlewheel is stable without coordinated apical molecules. Upon activation, a clear color change from cyan to dark blue occurs. Although the activation process implies also the loss of the guest molecules coordinated to the paddlewheel, the color change might not be a consequence of the reduced coordination number at $\mathrm{Cu}(\mathrm{II})$. In fact, the color darkening starts already at $\sim 50{ }^{\circ} \mathrm{C}$ when the chemisorbed molecules are not yet removed; see Figure SI 5. As a matter of fact, $\mathrm{Cu}$ (II) does not considerably change its electronic state, given that the linkage with the guest molecule occurs along a Jahn-Teller distorted direction (Table 2). The color change is rather associated with a modified refractive index and absorption when pores are filled.

In the filled and partially filled samples, the solvent present in the pores is heavily disordered, which increases the diffraction background (strongly affected also by the typical fluorescence of $\mathrm{Cu}$ stimulated by the $\mathrm{Mo} \mathrm{K} \alpha$ radiation we used). The disordered solvent molecules cannot be modeled, and therefore they produce large residual electron density peaks that affect the quality of refined models. The apical positions of the paddlewheel can be fully or partially occupied by coordinated $\mathrm{H}_{2} \mathrm{O}$ molecules. The estimated chemisorption degree (CS) for single crystals activated via treatments (a), (b), and (c) described in the MOF activation section are $55.8(23) \%, 28.6(9) \%$, and $14.3(6) \%$, respectively, in the approximation that the as-synthesized sample has $\mathrm{CS}=$ 
Table 2. Selected Bond Critical Point Properties ${ }^{a}$

\begin{tabular}{|c|c|c|c|c|c|}
\hline bond & property & $\mathrm{Cu}_{2}\left(\mathrm{H}_{2} \mathrm{BTC}\right)_{4}$ & $\mathrm{Cu}_{2}\left(\mathrm{H}_{2} \mathrm{BTC}\right)_{4}\left(\mathrm{H}_{2} \mathrm{O}\right)_{2}$ & $\mathrm{Zn}_{2}\left(\mathrm{H}_{2} \mathrm{BTC}\right)_{4}$ & $\mathrm{Zn}_{2}\left(\mathrm{H}_{2} \mathrm{BTC}\right)_{4}\left(\mathrm{H}_{2} \mathrm{O}\right)_{2}$ \\
\hline \multirow[t]{6}{*}{$\mathrm{M}-\mathrm{M}$} & $d(\AA)$ & 2.4622 & 2.5576 & 2.6446 & 2.9194 \\
\hline & $\rho_{\mathrm{bcp}}$ & 0.033 & 0.027 & 0.017 & 0.008 \\
\hline & $\nabla^{2} \rho_{\mathrm{bcp}}$ & 0.099 & 0.086 & 0.075 & 0.046 \\
\hline & DI & 0.131 & 0.110 & 0.054 & 0.019 \\
\hline & $|V| / G$ & 1.267 & 1.166 & 0.944 & 0.635 \\
\hline & $-K$ & -0.0090 & -0.0043 & -0.0010 & -0.0030 \\
\hline \multirow[t]{6}{*}{$\mathrm{M}-\mathrm{OCO}$} & $d(\AA)$ & 1.9461 & 1.9787 & 1.9958 & $1.9872 / 2.1049$ \\
\hline & $\rho_{\mathrm{bcp}}$ & 0.088 & 0.080 & 0.078 & $0.081 / 0.068$ \\
\hline & $\nabla^{2} \rho_{\text {bcp }}$ & 0.467 & 0.421 & 0.380 & $0.396 / 0.324$ \\
\hline & DI & 0.444 & 0.402 & 0.351 & $0.346 / 0.304$ \\
\hline & $|V| / G$ & 1.084 & 1.082 & 1.111 & $1.113 / 1.104$ \\
\hline & $-K$ & -0.0112 & -0.0089 & -0.0119 & $-0.0126 /-0.0095$ \\
\hline \multirow[t]{6}{*}{$\mathrm{M}-\mathrm{OH}_{2}$} & $d(\AA)$ & & 2.2359 & & 2.1023 \\
\hline & $\rho_{\mathrm{bcp}}$ & & 0.046 & & 0.060 \\
\hline & $\nabla^{2} \rho_{\text {bcp }}$ & & 0.189 & & 0.271 \\
\hline & DI & & 0.216 & & 0.263 \\
\hline & $|V| / G$ & & 1.112 & & 1.115 \\
\hline & $-K$ & & -0.0061 & & -0.0088 \\
\hline
\end{tabular}

${ }^{a} \rho_{\text {bcp }}$ and $\nabla^{2} \rho_{\text {bcp }}$ are the electron density $\left(e /\right.$ bohr $\left.^{3}\right)$ and its Laplacian $\left(e / b_{o h r^{5}}\right)$ at the bond critical point. DI is the delocalization index (electron pairs), $|V| / G$ is the ratio between potential and kinetic energy densities at the bond critical points, and $-K$ is the total bond energy density at the bond critical point (hartree/bohr ${ }^{3}$ ). The optimized fragments are reported in Figure SI_7.

$100 \%$. The progressively lower percentage of coordinated apical molecule implies a gradual and continuous shortening of the $\mathrm{Cu}-\mathrm{Cu}$ distance in the paddlewheel and related change of the carboxylate geometry; see Figure 4. The carboxylate

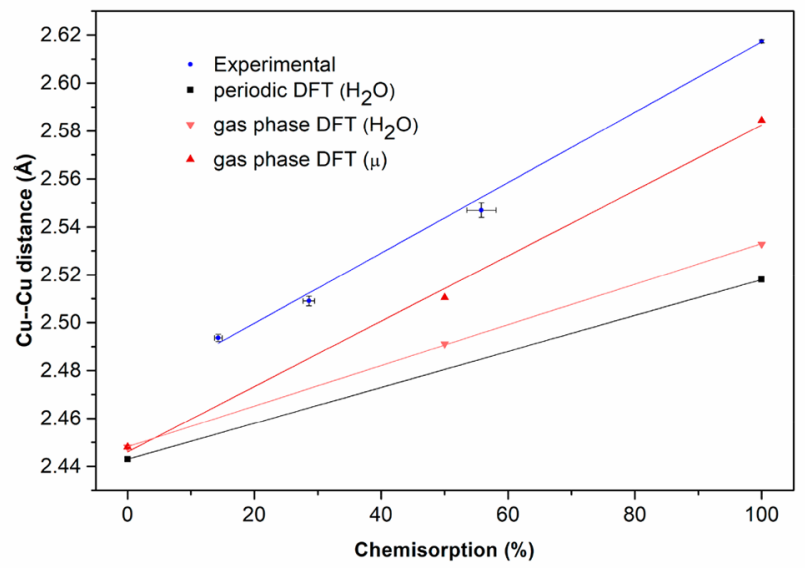

Figure 4. Dependence of $\mathrm{Cu}-\mathrm{Cu}$ distance in the paddlewheel as a function of coordinated apical $\mathrm{H}_{2} \mathrm{O}$ molecules from experiments as well as from geometry optimizations with zero, one, or two chemisorbed water molecules or dipole moments in their place.

coordination is ideal when there are no molecules apically coordinated to the paddlewheel. Instead, the extension of $\mathrm{Cu}-$ $\mathrm{Cu}$ distance increases the $\mathrm{O}-\mathrm{C}-\mathrm{O}$ and $\mathrm{C}-\mathrm{O}-\mathrm{Cu}$ angles and perturbs the $\mathrm{COO}-\mathrm{Cu}$ bonding. The structures refined for $\mathrm{CS}$ $=100 \%$ and $55.8(23) \%$ represent models for paddlewheels coordinated at both apical extremities or only at one, respectively. Of course, one cannot exclude for $\mathrm{CS}=$ $55.8(23) \%$ the disorder between doubly coordinated and naked paddlewheels. Instead, for CS $=28.6(9) \%$ or $14.3(6) \%$, the occupancy is certainly the result of disorder between different numbers of singularly (or doubly) coordinated and uncoordinated paddlewheel nodes. However, due to the very small difference between the atomic positions of the two limiting geometries (doubly and uncoordinated paddlewheel), it is not possible to refine two sets of coordinates and therefore a disordered model. For such a short atomic displacement (ca. $0.1 \AA$ ), a very high diffraction resolution would be necessary. ${ }^{46}$ DFT calculations predict well the trend of the paddlewheel geometry; however, the absolute values of $\mathrm{Cu}-\mathrm{Cu}$ distances strongly depend on the level of theory employed. The problem was already discussed in literature, either using copper formate paddlewheel $^{47,48}$ as a model for in vacuo calculations or simulating a whole crystal using periodic DFT. ${ }^{49}$ The latter reports $\mathrm{Cu}-\mathrm{Cu}$ distances that match very well our experimental findings.

The main interaction between guests and framework occurs at the paddlewheel, the geometry of which is deformed upon water coordination. The electronic structure of $\mathrm{Cu}$ atom is unaffected by the apical coordination ( $z$ direction), as it remains a $\mathrm{d}^{9}$ metal with $\mathrm{d}_{x^{2} y^{2}}$ half-occupied orbital. Thus, the distortion is not caused by the stereochemistry at the metal; see Table 2. We may therefore consider the role of electrostatics. As a matter of fact, the $\mathrm{M}-\mathrm{M}$ elongation is well reproduced by fixing a point dipole $(\mu=1.85 \mathrm{D})$ in place of the apical ligand (Figure 4). Indeed, the electrostatic interaction between the dipole moment and the paddlewheel causes qualitatively the same polarization induced by a water molecule, as revealed by electron density differences $[\rho$ (pw $\left.\left.+2 \mathrm{H}_{2} \mathrm{O}\right)-\rho(\mathrm{pw})-2 \mathrm{H}_{2} \mathrm{O}\right]$ and $[\rho(\mathrm{pw}+2 \mathrm{dip})-\rho(\mathrm{pw})](\mathrm{pw}$ $=$ paddlewheel) at fixed geometry; see Figure 5 . The apical interaction is therefore mainly electrostatic in nature, ${ }^{50}$ favored by the interplay between the negative site of the water molecule (the $\mathrm{O}$ atom) and the positively charged $\mathrm{Cu}$ atom, despite the filled $\mathrm{d}_{z}{ }^{2}$ orbital on the metal pointing toward the ligand and therefore hampering a genuine coordination. The ratio between potential and kinetic energy densities at the bond critical points $(|V| / G)$ as well as the total energy density $(-K)$ were calculated for the interaction between ligands and metals; see Table $2 .^{51-53}$ The correlation between $\mathrm{Cu}-\mathrm{Cu}$ distance (hence the CS degree) and bond critical point 


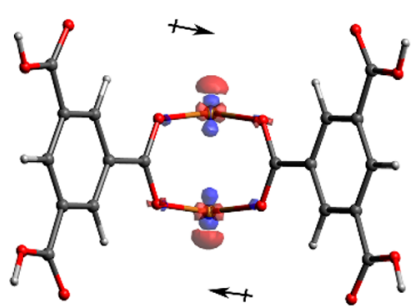

a

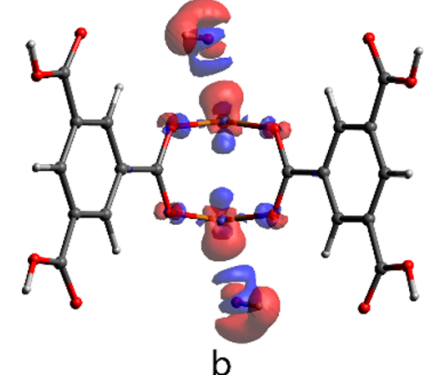

b
Figure 5. Difference of electron density: (a) $[\rho$ (pw+2dip) $-\rho(\mathrm{pw})]$ and $(\mathrm{b})\left[\rho\left(\mathrm{pw}+2 \mathrm{H}_{2} \mathrm{O}\right)-\rho(\mathrm{pw})-2 \mathrm{H}_{2} \mathrm{O}\right]$. Blue and red isosurfaces $\left( \pm 0.002 \mathrm{e} / \AA^{3}\right)$ correspond to positive and negative regions, respectively.

descriptors is linear, showing that the weakening of the $\mathrm{Cu}-$ $\mathrm{Cu}$ interaction increases the closed-shell character of this interaction (see Figure SI 9). The water dipole is so strong that it attracts the $\mathrm{Cu}$ atom and therefore elongates the $\mathrm{M}-\mathrm{M}$ bond. The absence of an orbital effect for this elongation is evident from the analysis of the deformation density in the region between metals: the polarization induced by the apical ligand induces charge accumulation in the $\mathrm{M}-\mathrm{M}$ region, but this excess of electron density cannot contribute to strengthen the $\mathrm{M}-\mathrm{M}$ interaction because it implies further accumulation in $\mathrm{d}_{z}{ }^{2}$ orbitals, which destabilizes even more the $M-M$ bond. The same effect, although weaker, is predicted for the $\mathrm{Zn}$ (II) paddlewheel.

The comparison between topological analyses of electron densities of closed-shell fragments of $\mathbf{1}$ and $\mathbf{2}$ confirms that the bonds between metals and carboxylate groups are stronger for $\mathrm{Cu}$ (II) than for $\mathrm{Zn}$ (II), as evident from the distances, $\rho_{\text {bcp }}$, and DI in Table 2. In agreement, calculations of the formation energy for the interaction between the carboxylate moiety and the paddlewheel of $\mathrm{Cu}(\mathrm{II})$ or $\mathrm{Zn}$ (II) retrieve stabilization energies of ca. -7.95 and $-7.80 \mathrm{eV}$, respectively. This is a consequence of the different stereoelectronics at the metals, $\mathrm{Cu}(\mathrm{II})$ being open shell and therefore with an orbital semioccupied and prompt for accepting donation from the carboxylate groups, whereas $\mathrm{Zn}$ (II) is a closed-shell metal, which can only guarantee electrostatic interactions with all ligands. Accordingly, the Laplacian of the electron density of 1 shows a region of charge concentration and depletion, whereas in $\mathbf{2}$ it is quite spherical (Figure 6). These features justify the lower dielectric constant calculated for 2 . The Laplacian maps

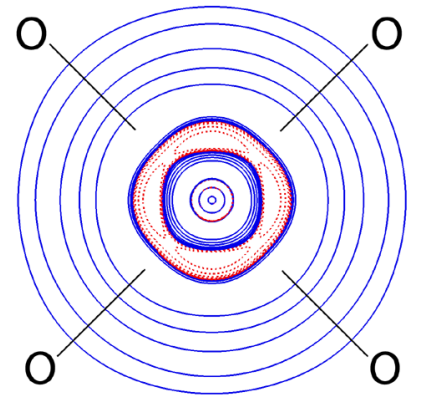

a

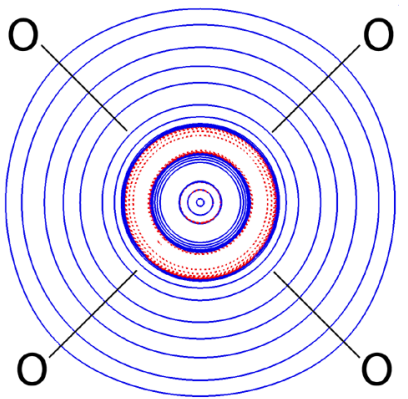

b
Figure 6. Laplacian at the metal site in the plane of bonding with the carboxylate groups: (a) Cu-based paddlewheel and (b) Zn-based paddlewheel. for $\mathbf{1}$ and $\mathbf{2}$ show instead similar features along the M-apical $\mathrm{H}_{2} \mathrm{O}$ interaction, along which the Laplacian does not show charge concentrations or depletions (Figure SI_11).

Despite the considerable change of the paddlewheel geometry between tetra- and penta-coordination of the metal, the atomic polarizability of the metal is only marginally affected; see Table 1 . Therefore, calculations of static- $\varepsilon_{\mathrm{r}}$ of frameworks with and without apical water molecules return values that do not differ considerably. Nevertheless, when water is coordinated, the framework displays a slightly larger static dielectric constant due to the additional electrons (and therefore electric polarizability) of the coordinated guests.

TGA measurements enable one to extract the amount of water molecules, estimated from the weight loss. For filled and partially filled materials, these estimations return 240 and 96 molecules per unit cell, respectively. Of these, 48 molecules per cell can occupy chemisorption sites. The calculated static polarizability of the coordinated water is $7.4 \mathrm{bohr}^{3}$, which is similar to the electronic polarizability obtained for an isolated $\mathrm{H}_{2} \mathrm{O}$ molecule $\left(6.9 \mathrm{bohr}^{3}\right)$. In the limit of additivity, the dielectric contribution of guest molecules can be explicated from the total $\varepsilon_{\mathrm{r}}$ and that of the empty framework by the Lorenz-Lorenz equation. ${ }^{54}$ Thereafter, the average polarizability of guest water molecules $\left(\left\langle\alpha_{\text {ads }}\right\rangle\right)$ was estimated in the high frequency regime $\left(10^{6} \mathrm{~Hz}\right)$ with the Clausius-Mossotti relation.

For 1 filled and partially filled, $\left\langle\alpha_{\text {ads }}\right\rangle$ is 78 and $200 \mathrm{bohr}^{3}$, respectively. These values can be compared to the estimated water molecule polarizabilities obtained from experimental dielectric constant of 100.0 for liquid water at $20^{\circ} \mathrm{C}$ and $10^{6}$ $\mathrm{Hz}\left(45\right.$ bohr $\left.^{3}\right)$; see Figure SI_8. It emerges that the mobility of water molecules hosted in the pores of $\mathbf{1}$ is emphasized thanks to the larger portion of volume available to them. The estimated values are significantly larger than those of the static molecular polarizability of isolated or coordinated water.

The calculated electrostatic potential inside the framework pores shows that the most positive site coincides in fact with the apical position of the paddlewheel, making it the most appealing site for coordination of the nucleophilic part of the guest molecules ( $\mathrm{O}$ atoms in water molecules), Figure 7. The strength of this coordination was estimated from the energy difference among the optimized geometries calculated with

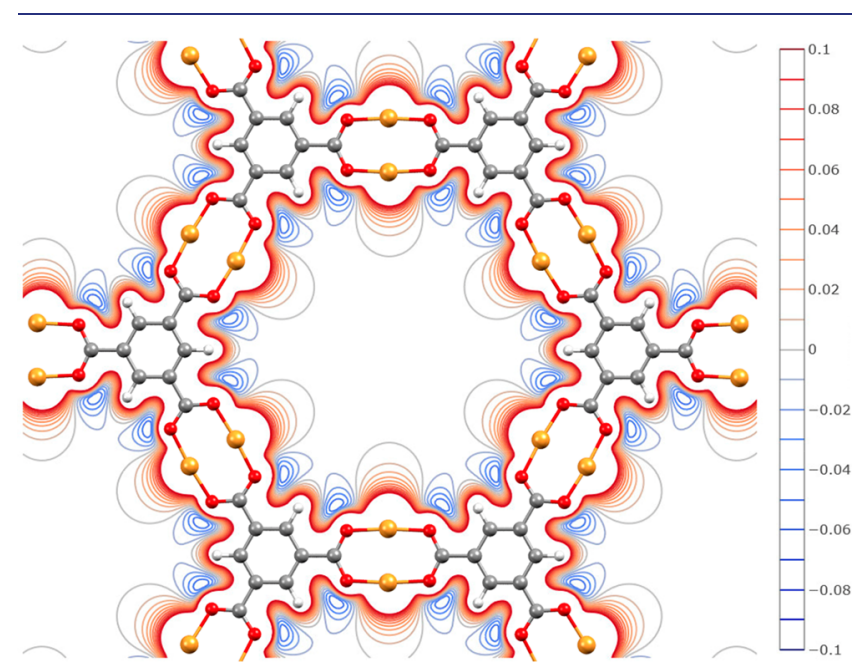

Figure 7. Electrostatic potential in the plane 111 for 1; contour levels are in atomic unit. 
either periodic-DFT or gas-phase DFT of paddlewheel, water molecule, and paddlewheel with chemisorbed water molecules. Depending on the kind of calculation, the obtained range is $50-65 \mathrm{~kJ} / \mathrm{mol}$, which classifies this interaction as a weak chemisorption (just above the classical limit of $0.5 \mathrm{eV}$, or 48 $\mathrm{kJ} / \mathrm{mol}$ ), although, as discussed above, the nature of the interaction is strongly dictated by electrostatics and therefore not enormously different from that of physisorption through a hydrogen bond. Sites where negative charge is in excess are, instead, identified close to $\mathrm{O}$ atoms of the carboxylate groups, whereas the $\mathrm{H}$ terminations of the aromatic rings are mildly positive. The negative charge sites are suitable for accepting $\mathrm{H}$ bonds from guest molecules. Because this interaction is weaker (and without any orbital contribution), the attachment to these sites is mainly a physisorption. The analysis of the bare framework does not allow one to identify further sites where guest molecules interact with each other producing an indirect sorption to the framework (via the chemi- or physisorbed molecules). These molecules are intrinsically less organized and therefore can be more mobile, thus mostly contributing to the dielectric constant.

\section{CONCLUSIONS}

In this work, we have investigated the charge distribution and the dielectric behavior of a highly porous and well-known MOF-type, $\mathrm{M}_{3}(\mathrm{BTC})_{2} \cdot$ (solvent) $)_{x}$, in view of potential application of these compounds as low-dielectric constant materials. The combination of theoretical simulations, X-ray diffraction, thermal gravimetry, and impedance measurements enables us to draw the following conclusions:

(a) Empty MOFs are indeed low dielectric constant materials and insensitive to the field frequency, mainly because of the large amount of unoccupied and unpolarizable volume inside the framework.

(b) Guest molecules are easily incorporated in the framework, during the synthesis or exposure of these materials to noninert atmosphere. These parasitic molecules significantly perturb the poorly polarizable volume and enhance the low and high frequency values of the dielectric constant.

(c) There are three different regimes of sorption, clearly identified by impedance spectroscopy: (1) chemisorption, due to molecules coordinated to the metal paddlewheel SBU, although this interaction remains mostly electrostatic; (2) physisorption, due to molecules hydrogen-bonded to the internal surfaces of the MOFs (thanks to specific binding sites); and (3) mobile molecules in the channels, which are not sorbed on the internal surfaces of the framework.

(d) The degree of chemisorption induces also some modifications to the framework, especially the coordination at apical free sites of the paddlewheels. Although a strict correlation exists between, for example, $\mathrm{M}-\mathrm{M}$ distances and the apical coordination, the static dielectric properties of the framework are almost unchanged.

This demonstrates the enhanced information on guest binding modes available when the dielectric properties are coupled with the information on the static electric field generated by the MOF. We believe that this study may foster further investigations combining $\mathrm{X}$-ray diffraction and impedance spectroscopy, to better depict the charge distribution and polarizability inside porous MOFs, with the aim to identify the key features of materials suitable for fabricating insulating devices necessary for microelectronics.

\section{ASSOCIATED CONTENT}

\section{Supporting Information}

The Supporting Information is available free of charge on the ACS Publications website at DOI: 10.1021/jacs.9b03643.

TGA curves, analysis of single-crystal color as a function of temperature, crystallographic information, DFT geometry optimizations as a function of the basis set, atomic polarizabilities, frequency-dependent experimental dielectric constant of water, bond critical point descriptors as a function of $\mathrm{Cu}-\mathrm{Cu}$ distance, chemical bonding analysis of a hydrogen-bonded guest molecule, Laplacian of the electron density at the metal site, and $3 \mathrm{D}$ electrostatic potential for $\mathrm{Cu}_{3}(\mathrm{BTC})_{2}$ (PDF)

$\mathrm{X}$-ray crystallographic data (ZIP)

\section{AUTHOR INFORMATION}

\section{Corresponding Authors}

*rebecca.scatena@dcb.unibe.ch

*piero.macchi@polimi.it

ORCID

Piero Macchi: 0000-0001-6292-9825

Present Address

${ }^{\S}$ Laboratory of Nanochemistry for Energy, EPFL, Rue de l'Industrie 17, Sion 1951, Switzerland.

\section{Notes}

The authors declare no competing financial interest.

\section{ACKNOWLEDGMENTS}

The Swiss National Science foundation has supported this research in the initial stages (project no. 160157). The National Competence Center for Research MARVEL is acknowledged for paying for the completion of this research.

\section{REFERENCES}

(1) Bersuker, I. B. The Jahn-Teller and Pseudo Jahn-Teller Effect in Materials Science. J. Phys.: Conf. Ser. 2017, 833 (1), No. 012001.

(2) Usman, M.; Mendiratta, S.; Lu, K.-L. Metal-Organic Frameworks: New Interlayer Dielectric Materials. ChemElectroChem 2015, 2 (6), 786-788.

(3) Furukawa, H.; Cordova, K. E.; O’Keeffe, M.; Yaghi, O. M. The Chemistry and Applications of Metal-Organic Frameworks. Science (Washington, DC, U. S.) 2013, 341 (6149), 1230444-1230444.

(4) Taddei, M.; Steitz, D. A.; van Bokhoven, J. A.; Ranocchiari, M. Continuous-Flow Microwave Synthesis of Metal-Organic Frameworks: A Highly Efficient Method for Large-Scale Production. Chem. Eur. J. 2016, 22 (10), 3245-3249.

(5) Khan, N. A.; Jhung, S. H. Synthesis of Metal-Organic Frameworks (MOFs) with Microwave or Ultrasound: Rapid Reaction, Phase-Selectivity, and Size Reduction. Coord. Chem. Rev. 2015, 285, $11-23$.

(6) Ockwig, N. W.; Delgado-Friedrichs, O.; O'Keeffe, M.; Yaghi, O. M. Reticular Chemistry: Occurrence and Taxonomy of Nets and Grammar for the Design of Frameworks. Acc. Chem. Res. 2005, 38 (3), 176-182.

(7) Yaghi, O. M.; O’Keeffe, M.; Ockwig, N. W.; Chae, H. K.; Eddaoudi, M.; Kim, J. Reticular Synthesis and the Design of New Materials. Nature 2003, 423 (6941), 705-714.

(8) Zagorodniy, K.; Seifert, G.; Hermann, H. Metal-Organic Frameworks as Promising Candidates for Future Ultralow-k Dielectrics. Appl. Phys. Lett. 2010, 97 (25), 251905.

(9) Jain, P.; Ramachandran, V.; Clark, R. J.; Zhou, H. D.; Toby, B. H.; Dalal, N. S.; Kroto, H. W.; Cheetham, A. K. Multiferroic Behavior Associated with an Order-Disorder Hydrogen Bonding Transition in 
Metal-Organic Frameworks (MOFs) with the Perovskite ABX 3 Architecture. J. Am. Chem. Soc. 2009, 131 (38), 13625-13627.

(10) Fu, D.-W.; Ge, J.-Z.; Dai, J.; Ye, H.-Y.; Qu, Z.-R. Synthesis, Structure and Dielectric Constant Properties of a Novel 1D Coordination Polymer[K(2-PTA)(H2O)]N. Inorg. Chem. Commun. 2009, 12 (10), 994-997.

(11) Fu, D.-W.; Dai, J.; Ge, J.-Z.; Ye, H.-Y.; Qu, Z.-R. Synthesis, Structure and Dielectric Properties of the First 1D Ba-Tetrazole Complex $[\mathrm{Ba}(4-\mathrm{TPA}) 2(\mathrm{H} 2 \mathrm{O}) 4 \cdot 3.5(\mathrm{H} 2 \mathrm{O})] \mathrm{N}$. Inorg. Chem. Commun. 2010, 13 (2), 282-285.

(12) Xu, R.-J.; Fu, D.-W.; Dai, J.; Zhang, Y.; Ge, J.-Z.; Ye, H.-Y. Synthesis, Structure and Dielectric Properties of Two Cr(III)Tetrazole Complexes Cr2(2-TPY)4(HO)2 and Cr(2-TPY)2(H2O)Cl. Inorg. Chem. Commun. 2011, 14 (7), 1093-1096.

(13) Sánchez-Andújar, M.; Yáñez-Vilar, S.; Pato-Doldán, B.; GómezAguirre, C.; Castro-García, S.; Señarís-Rodríguez, M. A. Apparent Colossal Dielectric Constants in Nanoporous Metal Organic Frameworks. J. Phys. Chem. C 2012, 116 (24), 13026-13032.

(14) Chen, Q.; Guo, P.-C.; Zhao, S.-P.; Liu, J.-L.; Ren, X.-M. A Rhombus Channel Metal-Organic Framework Comprised of Sr2+ and Thiophene-2, 5-Dicarboxylic Acid Exhibiting Novel Dielectric Bistability. CrystEngComm 2013, 15 (6), 1264.

(15) Usman, M.; Lee, C. H.; Hung, D. S.; Lee, S. F.; Wang, C. C.; Luo, T. T.; Zhao, L.; Wu, M. K.; Lu, K. L. Intrinsic Low Dielectric Behaviour of a Highly Thermally Stable Sr-Based Metal-Organic Framework for Interlayer Dielectric Materials. J. Mater. Chem. C 2014, 2, 3762-3768.

(16) Mendiratta, S.; Usman, M.; Luo, T.; Chang, B.; Lee, S.; Lin, Y.; Lu, K. Anion-Controlled Dielectric Behavior of Homochiral Tryptophan-Based Metal-Organic Frameworks. Cryst. Growth Des. 2014, 14 (4), 1572-1579.

(17) Mendiratta, S.; Usman, M.; Luo, T.-T.; Lee, S.-F.; Lin, Y.-C.; Lu, K.-L. Guest Dependent Dielectric Properties of Nickel(II)-Based Supramolecular Networks. CrystEngComm 2014, 16 (28), 63096315.

(18) Kchaou, H.; Karoui, K.; Khirouni, K.; Ben Rhaiem, A. Optical and Dielectric Relaxation of Transition Metal-Based OrganicInorganic Hybrid Materials. J. Alloys Compd. 2017, 728, 936-943.

(19) Ryder, M. R.; Zeng, Z.; Titov, K.; Sun, Y.; Mahdi, E. M.; Flyagina, I.; Bennett, T. D.; Civalleri, B.; Kelley, C. S.; Frogley, M. D.; Cinque, G.; Tan, J.-C. Dielectric Properties of Zeolitic Imidazolate Frameworks in the Broad-Band Infrared Regime. J. Phys. Chem. Lett. 2018, 9 (10), 2678-2684.

(20) Warmbier, R.; Quandt, A.; Seifert, G. Dielectric Properties of Selected Metal-Organic Frameworks. J. Phys. Chem. C 2014, 118 (22), 11799-11805.

(21) Ernst, M.; Dos Santos, L. H. R.; Macchi, P. Optical Properties of Metal Organic Networks from Distributed Atomic Polarizabilities. CrystEngComm 2016, 18, 7339-7346.

(22) Ryder, M. R.; Donà, L.; Vitillo, J. G.; Civalleri, B. Understanding and Controlling the Dielectric Response of MetalOrganic Frameworks. ChemPlusChem 2018, 83 (4), 308-316.

(23) Chui, S. S.-Y. A Chemically Functionalizable Nanoporous Material [Cu3(TMA)2(H2O)3]N. Science (Washington, DC, U. S.) 1999, 283 (5405), 1148-1150.

(24) Redel, E.; Wang, Z.; Walheim, S.; Liu, J.; Gliemann, H.; Wöll, C. On the Dielectric and Optical Properties of Surface-Anchored Metal-Organic Frameworks: A Study on Epitaxially Grown Thin Films. Appl. Phys. Lett. 2013, 103 (9), 0-5.

(25) Poulsen, R. D.; Bentien, A.; Chevalier, M.; Iversen, B. B. Synthesis, Physical Properties, Multitemperature Crystal Structure, and $20 \mathrm{~K}$ Synchrotron X-Ray Charge Density of a Magnetic Metal Organic Framework Structure, Mn 3 (C 8 O 4 H 4) 3 (C 5 H 11 ON) 2. J. Am. Chem. Soc. 2005, 127 (25), 9156-9166.

(26) Poulsen, R. D.; Jørgensen, M. R. V.; Overgaard, J.; Larsen, F. K.; Morgenroth, W.; Graber, T.; Chen, Y.-S.; Iversen, B. B. Synchrotron X-Ray Charge-Density Study of Coordination Polymer $[\mathrm{Mn}(\mathrm{HCOO}) 2(\mathrm{H} 2 \mathrm{O}) 2] \infty$. Chem. - Eur. J. 2007, 13 (35), 97759790.
(27) Dos Santos, L. H. R.; Lanza, A.; Barton, A. M.; Brambleby, J.; Blackmore, W. J. A.; Goddard, P. A.; Xiao, F.; Williams, R. C.; Lancaster, T.; Pratt, F. L.; Blundell, S. J.; Singleton, J.; Manson, J. L.; Macchi, P. Experimental and Theoretical Electron Density Analysis of Copper Pyrazine Nitrate Quasi-Low-Dimensional Quantum Magnets. J. Am. Chem. Soc. 2016, 138 (7), 2280-2291.

(28) Kubus, M.; Lanza, A.; Scatena, R.; Dos Santos, L. H. R.; Wehinger, B.; Casati, N.; Fiolka, C.; Keller, L.; Macchi, P.; Rüegg, C.; Krämer, K. W. Quasi-2D Heisenberg Antiferromagnets [CuX $\left.(\mathrm{Pyz})_{2}\right]$ $\left(\mathrm{BF}_{4}\right)$ with $\mathrm{X}=\mathrm{Cl}$ and Br. Inorg. Chem. 2018, 57 (9), 4934-4943.

(29) Wu, Y.; Kobayashi, A.; Halder, G. J.; Peterson, V. K.; Chapman, K. W.; Lock, N.; Southon, P. D.; Kepert, C. J. Negative Thermal Expansion in the Metal-Organic Framework Material $\mathrm{Cu} 3$ (1,3,5Benzenetricarboxylate) 2. Angew. Chem., Int. Ed. 2008, 47 (46), 8929-8932.

(30) Feldblyum, J. I.; Liu, M.; Gidley, D. W.; Matzger, A. J. Reconciling the Discrepancies between Crystallographic Porosity and Guest Access As Exemplified by Zn-HKUST-1. J. Am. Chem. Soc. 2011, 133 (45), 18257-18263.

(31) Schlichte, K.; Kratzke, T.; Kaskel, S. Improved Synthesis, Thermal Stability and Catalytic Properties of the Metal-Organic Framework Compound Cu3(BTC)2. Microporous Mesoporous Mater. 2004, 73 (1-2), 81-88.

(32) Macchi, P.; Bürgi, H.-B.; Chimpri, A. S.; Hauser, J.; Gál, Z. Low-Energy Contamination of Mo Microsource X-Ray Radiation: Analysis and Solution of the Problem. J. Appl. Crystallogr. 2011, 44 (4), 763-771.

(33) CrysAlisPro, 2017.

(34) Sheldrick, G. M. A Short History of SHELX. Acta Crystallogr., Sect. A: Found. Crystallogr. 2008, 64 (1), 112-122.

(35) Cruickshank, D. W. J.; Rollett, J. S. Electron-Density Errors at Special Positions. Acta Crystallogr. 1956, 9 (2), 203-203.

(36) Rees, B. Variance and Covariance in Experimental Electron Density Studies, and the Use of Chemical Equivalence. Acta Crystallogr., Sect. A: Cryst. Phys., Diffr., Theor. Gen. Crystallogr. 1976, 32 (3), 483-488.

(37) Schmøkel, M. S.; Bjerg, L.; Larsen, F. K.; Overgaard, J.; Cenedese, S.; Christensen, M.; Madsen, G. K. H.; Gatti, C.; Nishibori, E.; Sugimoto, K.; Takata, M.; Iversen, B. B. Comparative Study of XRay Charge-Density Data on CoSb 3. Acta Crystallogr., Sect. A: Found. Crystallogr. 2013, 69 (6), 570-582.

(38) Dovesi, R.; Saunders, V. R.; Roetti, C.; Orlando, R.; ZicovichWilson, C. M.; Pascale, F.; Civalleri, B.; Doll, K.; Harrison, N. M.; Bush, I. J.; D’Arco, P.; Llunell, M.; Causà, M.; Noël, Y. CRYSTAL14 User's Manual; University of Torino: Torino, 2014.

(39) Peintinger, M. F.; Oliveira, D. V.; Bredow, T. Consistent Gaussian Basis Sets of Triple-Zeta Valence with Polarization Quality for Solid-State Calculations. J. Comput. Chem. 2013, 34 (6), 451-459.

(40) Frisch, M. J.; Trucks, G. W.; Schlegel, H. B.; Scuseria, G. E.; Robb, M. A.; Cheeseman, J. R.; Scalmani, G.; Barone, V.; Mennucci, B.; Petersson, H. N.; Caricato, M.; Li, X.; Hratchian, H. P.; Izmaylov, A. F.; Bloino, J.; Zheng, G.; Sonnenberg, J. L.; Hada, M.; Ehara, M.; et al. Gaussian 09; Gaussian, Inc.: Wallingford, CT, 2009.

(41) Keith, T. A. AIMAll. TK Gristmill Software; Overland Park: KS, 2017.

(42) Krawczuk, A.; Pérez, D.; Macchi, P. PolaBer: A Program to Calculate and Visualize Distributed Atomic Polarizabilities Based on Electron Density Partitioning. J. Appl. Crystallogr. 2014, 47 (4), $1452-1458$.

(43) Macrae, C. F.; Bruno, I. J.; Chisholm, J. A.; Edgington, P. R.; McCabe, P.; Pidcock, E.; Rodriguez-Monge, L.; Taylor, R.; Van De Streek, J.; Wood, P. A. Mercury CSD 2.0 - New Features for the Visualization and Investigation of Crystal Structures. J. Appl. Crystallogr. 2008, 41 (2), 466-470.

(44) Song, X.; Jeong, S.; Kim, D.; Lah, M. S. Transmetalations in Two Metal-Organic Frameworks with Different Framework Flexibilities: Kinetics and Core-Shell Heterostructure. CrystEngComm 2012, 14 (18), 5753. 
(45) Allen, F. H. The Cambridge Structural Database: A Quarter of a Million Crystal Structures and Rising. Acta Crystallogr., Sect. B: Struct. Sci. 2002, 58 (3 PART 1), 380-388.

(46) Bragg, W. L.; West, J. A Note on the Representation of Crystal Structure by Fourier Series. Philos. Mag. 1930, 10, 823.

(47) Tafipolsky, M.; Amirjalayer, S.; Schmid, R. First-PrinciplesDerived Force Field for Copper Paddle-Wheel-Based Metal-Organic Frameworks. J. Phys. Chem. C 2010, 114 (34), 14402-14409.

(48) Toda, J.; Fischer, M.; Jorge, M.; Gomes, J. R. B. Water Adsorption on a Copper Formate Paddlewheel Model of CuBTC: A Comparative MP2 and DFT Study. Chem. Phys. Lett. 2013, 587, 713.

(49) Watanabe, T.; Sholl, D. S. Molecular Chemisorption on Open Metal Sites in Cu3(Benzenetricarboxylate)2: A Spatially Periodic Density Functional Theory Study. J. Chem. Phys. 2010, 133 (9), No. 094509.

(50) Herich, P.; Bučinský, L.; Breza, M.; Gall, M.; Fronc, M.; Petřiček, V.; Kožíšek, J. Electronic Structure of Two Isostructural paddle-Wheel' Complexes: A Comparative Study. Acta Crystallogr., Sect. B: Struct. Sci., Cryst. Eng. Mater. 2018, 74 (6), 681-692.

(51) Espinosa, E.; Alkorta, I.; Rozas, I.; Elguero, J.; Molins, E. About the Evaluation of the Local Kinetic, Potential and Total Energy Densities in Closed-Shell Interactions. Chem. Phys. Lett. 2001, 336 (5-6), 457-461.

(52) Macchi, P.; Sironi, A. Chemical Bonding in Transition Metal Carbonyl Clusters: Complementary Analysis of Theoretical and Experimental Electron Densities. Coord. Chem. Rev. 2003, 238-239, 383-412.

(53) Gatti, C. Chemical Bonding in Crystals: New Directions. Z. Kristallogr. - Cryst. Mater. 2005, 220 (5/6), 399.

(54) Heller, W. Remarks on Refractive Index Mixture Rules. J. Phys. Chem. 1965, 69 (4), 1123-1129. 